\title{
Assessing the quality of scanning probe microscope designs
}

\author{
James B Thompson, Barney Drake, Johannes H Kindt, \\ Jessica Hoskins and Paul K Hansma \\ Department of Physics, University of California, Santa Barbara, CA 93106, USA \\ E-mail: jbthomp@physics.ucsb.edu
}

Received 8 June 2001, in final form 3 August 2001

Published 28 August 2001

Online at stacks.iop.org/Nano/12/394

\begin{abstract}
We present a method for assessing an atomic force microscope's (AFM's) ability to reject externally applied vibrations. This method is demonstrated on one commercial and two prototype AFMs. For optimally functioning AFMs, we find that the response to externally applied vibrations obeys a $1 / \omega^{2}$ frequency dependence. This $1 / \omega^{2}$ frequency dependence can be understood by modelling the mechanical system which connects the AFM cantilever and the sample under test as a simple harmonic oscillator.

According to such a model, the resonant frequency of the mechanical system which connects the AFM cantilever and the sample under test determines an AFM's ability to reject externally applied, low-frequency vibrations.
\end{abstract}

\section{Introduction}

The atomic force microscope (AFM) measures surface topography, or force, by monitoring the deflection of a micromachined cantilever beam (Binnig 1986, Albrecht and Quate 1988). AFMs enable the measurement of subnanometre features and sub-nanonewton forces (Weisenhorn et al 1989, Rugar and Hansma 1990, Hoh et al 1992, Ohnesorge and Binnig 1993, Lee et al 1994, Florin et al 1994, Stowe et al 1997, Giessibl et al 2000).

Typically, the force resolution of an AFM using an optical detection system (McClelland et al 1987, Meyer and Amer 1988, Alexander et al 1989) is limited by thermal cantilever vibrations, or noise in the optical detection system (Sarid 1991, Smith 1995). When low-spring-constant cantilevers are used, thermal cantilever vibrations dominate and optimal force resolution is achieved (Viani et al 1999). Beyond this, improvements in force resolution can be attained by either lowering the temperature of the experiment or reducing the cantilever's coefficient of viscous damping (Stowe et al 1997, Viani et al 1999).

The AFM is an important tool for studying biological systems under near-physiological conditions (Drake et al 1989, Hansma et al 1992, Mou et al 1996, Kasas et al 1997, Reif et al 1997, Oberhauser et al 1998, Merkel et al 1999, Oesterhelt et al 2000, Viani et al 2000). Such studies must be carried out in liquids and within a certain temperature range. Under these restrictions, the most immediate way to improve force resolution is to reduce the cantilever's size and, therefore, its coefficient of viscous damping (Walters et al 1996, Schaffer et al 1997, Viani et al 1999).

As the cantilevers are made smaller, the optical, electronic, and mechanical components of the AFM must improve so that they do not limit the AFM's overall noise performance.

Below we will examine how externally applied vibrations couple to the AFM deflection signal, demonstrate a technique to measure an AFM's ability to reject externally applied vibrations, discuss what design parameters make an AFM resistant to externally applied vibrations and show that our most recent small-cantilever AFM has mechanical characteristics as good as or better than those seen in commercial large-cantilever AFMs.

\section{Simple theoretical model}

The mechanical system connecting the AFM cantilever to the sample under study can be treated as a simple harmonic oscillator with a certain mass $m$, spring constant $k$ and damping factor $b$ (Michely et al 2000). The equation of motion for this system is

$$
m \frac{\mathrm{d}^{2} x}{\mathrm{~d} t^{2}}+b \frac{\mathrm{d} x}{\mathrm{~d} t}+k x=m \frac{\mathrm{d}^{2} v}{\mathrm{~d} t^{2}}
$$

where $x$ is the tip-sample distance and $v$ is the vibration applied to the instrument. Taking $v=A_{v} \mathrm{e}^{\mathrm{i} \omega t}$ and $x=A_{x} \mathrm{e}^{\mathrm{i} \omega t}$ we can 


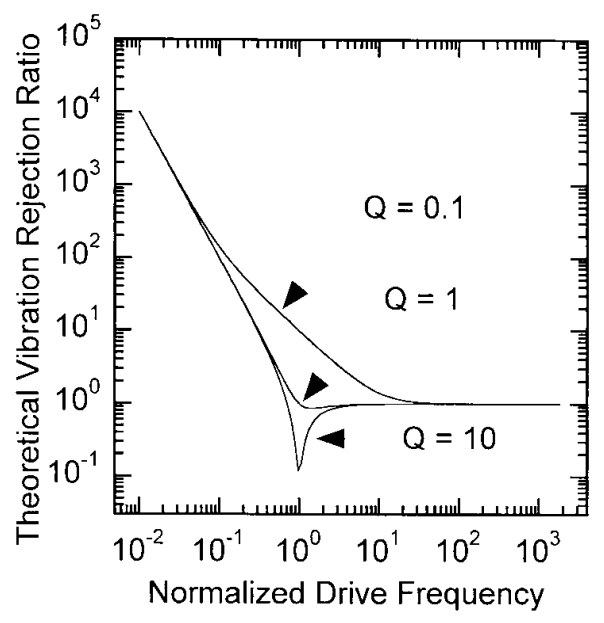

Figure 1. Theoretical vibration rejection ratio versus frequency. Curves are shown for $Q$ values of $0.1,1$ and 10. Vibration rejection ratio is not sensitive to $Q$ at low frequencies, but is sensitive to $Q$ near resonance.

find the complex vibration rejection ratio

$$
\frac{A_{v}}{A_{x}}=\left(1-\frac{\omega_{0}^{2}}{\omega^{2}}\right)-\mathrm{i} \frac{1}{Q} \frac{\omega_{0}}{\omega}
$$

where $\omega_{0}=(k / m)^{1 / 2}$ and $Q=b^{-1}(k m)^{1 / 2}$. The amplitude of this quantity is the vibration rejection ratio which is plotted in figure 1:

$$
\left|\frac{A_{v}}{A_{x}}\right|=\sqrt{\left(\frac{\omega_{0}^{2}}{\omega^{2}}-1\right)^{2}+\frac{1}{Q^{2}} \frac{\omega_{0}^{2}}{\omega^{2}}} .
$$

Vibration rejection ratio can be measured by comparing the applied vibration amplitude with the resulting cantilever deflection amplitude while the cantilever is held in contact with the sample surface. In the low-frequency limit $(\omega \ll$ $\omega_{0}$ ) this equation has a particularly simple form (Rugar and Hansma 1990)

$$
\left|\frac{A_{v}}{A_{x}}\right|=\frac{\omega_{0}^{2}}{\omega^{2}}+\vartheta\left(\frac{\omega_{0}}{\omega}\right) .
$$

Therefore, if the resonant frequency of the mechanical system connecting the cantilever to the sample is much lower than the applied vibration frequency, we should expect the vibration rejection ratio to have a $1 / \omega^{2}$ frequency dependence.

\section{Measuring vibration rejection ratio}

To measure the vibration rejection ratio of an AFM, it is necessary to introduce a known vibration into the AFM body and compare it with the amplitude of the cantilever deflection signal, see figure 2 . We introduced vibrations into the AFM body by placing the entire instrument onto a tactile sound transducer (Clark Synthesis). The tactile sound transducer was driven with the output of an audio amplifier (AudioSource), which in turn was driven with a sinusoidal signal (Wavetek function generator). The amplitude of the vibrations introduced into the AFM was measured with a fibre optic position sensor (MTI Systems). The output of

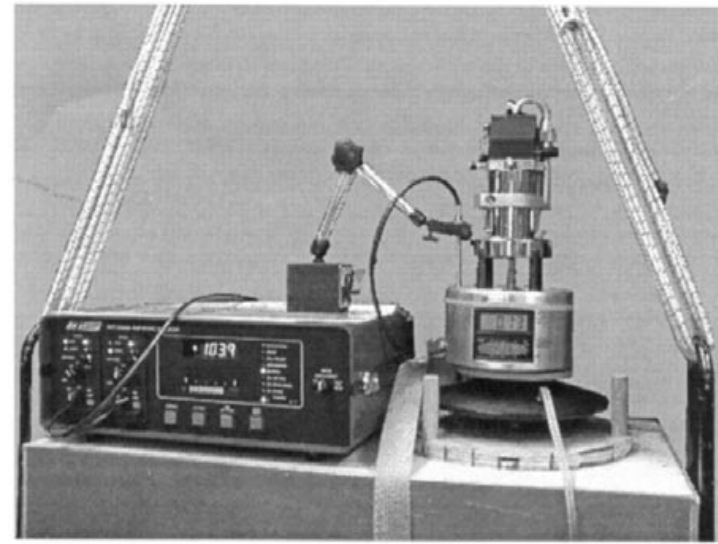

Figure 2. This test setup allows us to determine the response of an AFM to an externally applied vibration. A tactile sound transducer is used to vibrate the AFM. These vibrations are characterized using a fibre optic position sensor. By comparing the amplitude of the applied vibrations to the amplitude of the AFM deflection signal it is possible to determine an AFM's ability to reject spurious signals, such as room vibrations or sound.

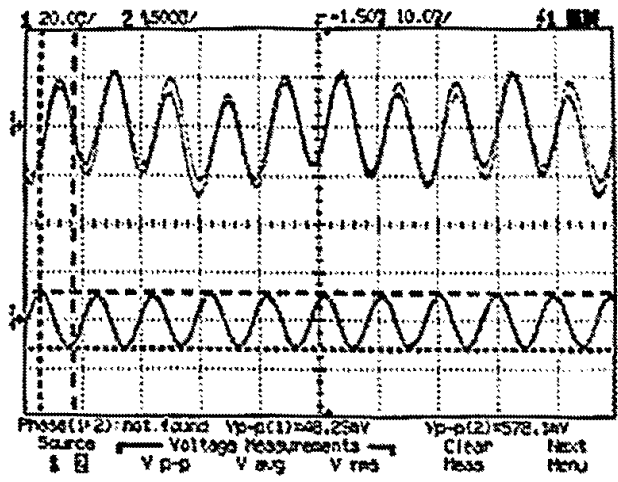

Figure 3. The response of a prototype, small-cantilever AFM to an applied vibration. The top trace is the applied vibration as measured by a fibre optic position sensor. The lower trace is the resulting deflection signal from the AFM.

the sensor was filtered and displayed on an oscilloscope for measurement. The fibre optic position sensor was calibrated before each set of data was taken. The deflection signal was obtained from our commercial AFM electronics using a Signal Access Module (all of the AFMs tested were designed to be pin compatible with standard NanoScope SPM electronics from Digital Instruments), amplified, and filtered before being displayed on an oscilloscope for measurement.

The vibration rejection ratio of an instrument was then measured using the following simple procedure: first the AFM tip and sample (freshly cleaved mica epoxied to a steel disc) were brought into contact within distilled water. Then a force curve was acquired in order to calibrate the deflection signal. The $z$-range of the force curve was set to zero, disabling the $z$ feedback and leaving the tip in hard contact with the surface. At this point, the frequency of the applied vibration was selected and the amplitude of the applied vibration chosen to produce a sinusoidal deflection signal of several nanometres. We used applied vibration amplitudes of $20 \mathrm{~nm}$ to $20 \mu \mathrm{m}$. Finally, the vibration amplitudes and deflection amplitudes were measured on the oscilloscope, see figure 3. Both measurements were 

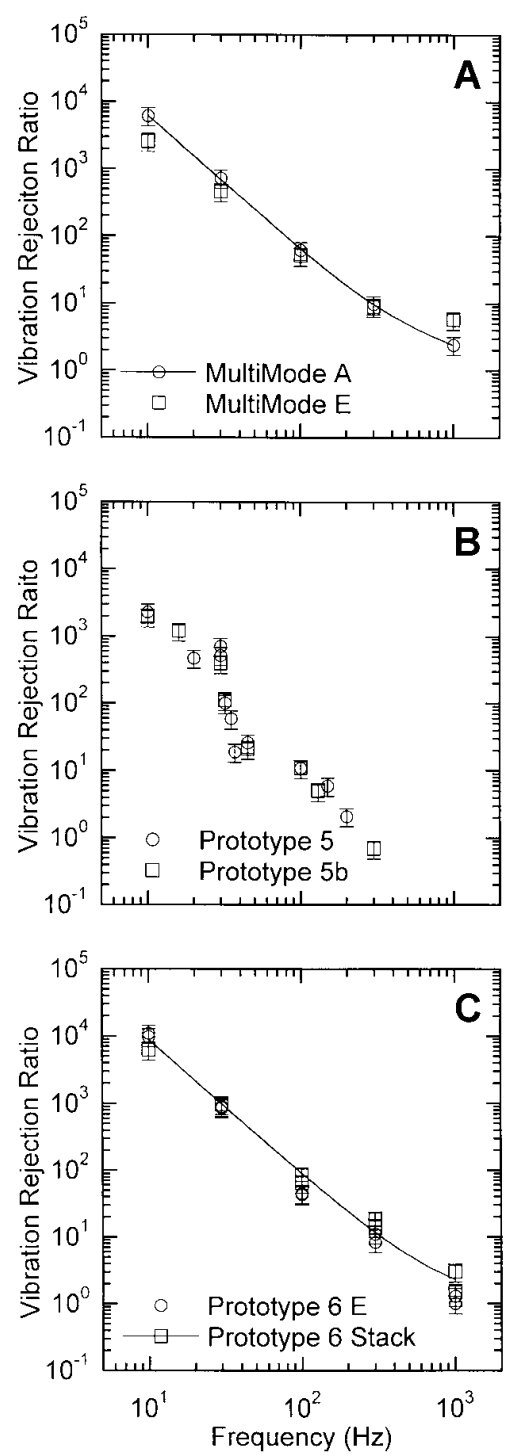

Figure 4. These plots show the vibration rejection ratios of three different AFMs (vibration rejection ratio is the amplitude of the applied vibration divided by the amplitude of the cantilever deflection it produces). A large vibration rejection ratio implies that the AFM is not sensitive to external noise sources such as room vibrations or sound. (a) These data were taken on a commercial AFM (MultiMode, Digital Instruments) with two different commercial piezoelectric-tube scanners. (b) These data were taken on a prototype, small-cantilever AFM before and after a design modification. No change in the instrument's vibration rejection ratio was observed. $(c)$ The performance of a more recent, prototype, small-cantilever AFM using a commercial piezoelectric-tube scanner and a custom scanner constructed from piezoelectric stacks. If an AFM's first mechanical resonance is well above the applied vibration frequency, then the vibration rejection ratio should be high and proportional to $1 / \omega^{2}$, where $\omega$ is the applied vibration frequency (see text). The solid curves shown in $(a)$ and $(c)$ are curve fits to equation (3) in the text. The vibration rejection ratio in $(a)$ appears to be proportional to $1 / \omega^{2}$ below $300 \mathrm{~Hz}$. The vibration rejection ratio in $(b)$, however, shows a more complicated dependence on $\omega$ suggesting that this AFM has one or more mechanical resonances below $300 \mathrm{~Hz}$. The vibration rejection ratios of the small-cantilever AFM shown in $(c)$ demonstrate a $1 / \omega^{2}$ dependence and the same or better mechanical performance (as measured by vibration rejection ratio) as that achieved in existing commercial AFMs. converted into nanometres, and the vibration rejection ratio was calculated by dividing the vibration amplitude by the deflection amplitude. Several measurements were made at different frequencies on each instrument.

\section{Results}

Three different AFMs were tested to determine their relative vibration rejection performance.

Figure 4(a) shows the vibration rejection performance of a commercial AFM (MultiMode, Digital Instruments) using a silicon nitride cantilever in water. In one case a commercial 'A' piezoelectric-tube scanner ( $1 \mu \mathrm{m}$ scan range) was used, and in the other a commercial ' $E$ ' piezoelectric-tube scanner (15 $\mu \mathrm{m}$ scan range). These two sets of data are similar, and in both cases the vibration rejection ratios show the expected $1 / \omega^{2}$ dependence up to $300 \mathrm{~Hz}$. The fit suggests that the first resonance of the mechanical system connecting the cantilever to the sample is about $800 \mathrm{~Hz}$.

The data in figure $4(b)$ were taken on a prototype, small-cantilever AFM. These data do not show a clear $1 / \omega^{2}$ dependence and the vibration rejection ratio values are lower at all frequencies. Clearly, the simple harmonic oscillator model does not adequately describe these data. As a result, we modified the design of this AFM in the hope of increasing its vibration rejection ratio, but no change in the vibration rejection ratio was observed after the modification. We are forced to conclude that our AFM prototype \#5 is flawed.

The data in figure 4(c) show the vibration rejection ratios measured on one of our latest prototype small-cantilever AFMs. This instrument's performance is much better than that shown in figure $4(b)$. The fit in figure $4(c)$ suggests that the first resonance of the mechanical system connecting the cantilever to the sample is about $950 \mathrm{~Hz}$. Data are shown for this AFM using both a commercial E piezoelectric-tube scanner and a custom piezoelectric-stack scanner $(6 \mu \mathrm{m}$ scan size). The measured vibration rejection ratios are similar when using either scanner, suggesting either that their mechanical performance is not different or that we are not currently limited by the design of the piezoelectric scanner used.

\section{Discussion and conclusion}

In optimally functioning AFMs, we observe the predicted $1 / \omega^{2}$ dependence in vibration rejection ratio. From our idealized model we can expect that the term in the instrument's vibration rejection ratio proportional to $1 / \omega^{2}$ depends only on the first resonant frequency of the mechanical system connecting the cantilever to the sample. Damping, for example, contributes a term proportional to $1 / \omega$. The observation of a $1 / \omega^{2}$ dependence supports our theory that the first resonant frequency of the mechanical system connecting the cantilever to the sample is of key importance to an AFM's ability to reject unwanted low-frequency noise sources, such as room vibrations or sound.

It should be possible to extend the model of AFM vibration rejection ratios presented here to AFMs whose performance is not optimal. To do so, the mechanical system connecting the cantilever to the sample could be modelled as two or more coupled harmonic oscillators, instead of one. However, a 
more complicated analysis than that presented is frequently unnecessary.

Given that increased damping does not improve an AFM's vibration rejection ratio at low frequency, the only clear way to improve an AFM's resistance to low-frequency vibrations is, not surprisingly, to eliminate low-frequency resonances. In order to achieve this, the AFM designer must either reduce the mass of the AFM head, or increase its stiffness. Further, the same logic applies to the design of any mechanical actuator or probe for use on the nanometre scale.

Finally, we conclude that a plot of vibration rejection ratio versus frequency is a simple but powerful tool to evaluate an AFM design.

\section{Acknowledgments}

This work was supported by the Materials Research Division of the National Science Foundation (grant number NSFDMR9988640), the Materials Research Laboratory Program of the National Science Foundation (grant number DMR96-32716) and the MURI programme of the Army Research Office (grant number DAAH04-96-1-0443).

\section{References}

Albrecht T and Quate C 1988 J. Vac. Sci. Technol. A 6 271-4 Alexander S, Hellemans L, Marti O, Schneir J, Elings V and Hansma P 1989 J. Appl. Phys. 65 164-7

Binnig G, Quate C and Gerber C 1986 Phys. Rev. Lett. 56 930-3

Drake B, Prater C, Weisenhorn A, Gould S, Albrecht T, Quate C, Cannell D, Hansma H and Hansma P 1989 Science 243 1586-8

Florin E, Moy V and Gaub H 1994 Science 264 415-7

Giessibl F, Hembacher S, Bielefeldt H and Mannhart J 2000 Science $289422-5$
Hansma H, Vesenka J, Siegerist C, Kelderman G, Morret H, Sinsheimer R, Elings V, Bustamante C and Hansma P 1992 Science 256 1180-4

Hoh J, Cleveland J, Prater C, Revel J and Hansma P 1992 J. Am. Chem. Soc. 1992 4917-8

Kasas S, Thompson N, Smith B, Hansma H, Zhu X, Guthold M, Bustamante C, Kool E, Kashlev M and Hansma P 1997 Biochem. 36 461-8

Lee G, Chrisey L and Colton R 1994 Science 266 771-3

McClelland G, Erlandsson R and Chiang S 1987 Review of Progress in Quantitative Nondestructive Evaluation ed D O Thompson and D E Chimenti (New York: Plenum)

Merkel R, Nassoy P, Leung A, Ritchie K and Evans E 1999 Nature 397 50-3

Meyer G and Amer N 1988 Appl. Phys. Lett. 53 2400-2

Michely T, Kaiser M and Rost M 2000 Rev. Sci. Instrum. 71 4461-7

Mou J, Sheng S, Ho R and Shao Z 1996 Biophys. J. 71 2213-21

Oberhauser A, Marszalek P, Erickson H and Fernandez J 1998 Nature 393 181-5

Oesterhelt F, Oesterhelt D, Pfeiffer M, Engel A, Gaub H and Muller D 2000 Science 288 143-6

Ohnesorge F and Binnig G 1993 Science 260 1451-6

Reif M, Gautel M, Oesterhelt F, Fernandez J and Gaub H 1997 Science 276 1109-12

Rugar D and Hansma P 1990 Phys. Today 43 23-30

Sarid D 1991 Scanning Force Microscopy (New York: Oxford University Press)

Shaffer T, Viani M, Walters D, Drake B, Runge E, Cleveland J, Wendman M and Hansma P 1997 Proc. SPIE 3009 48-52

Smith D 1995 Rev. Sci. Instrum. 66 3191-5

Stowe T, Yasumura K, Kenny T, Botkin D, Wago K and Rugar D 1997 Appl. Phys. Lett. 71 288-90

Viani M, Pietrasanta L, Thompson J, Chand A, Gebeshuber I, Kindt J, Richter M, Hansma H and Hansma P 2000 Nature Struct. Biol. 7 644-7

Viani M, Shaffer T, Chand A, Rief M, Gaub H and Hansma P 1999 J. Appl. Phys. 86 2258-62

Walters D, Cleveland J, Thompson N, Hansma P, Wendman M, Gurley G and Elings V 1996 Rev. Sci. Instrum. 67 3583-90

Weisenhorn A, Hansma P, Albrecht T and Quate C 1989 Appl. Phys. Lett. 54 2651-3 